\title{
APPLICATION OF LAGRANGE MULTIPLIER METHOD FOR COMPUTING FOLD BIFURCATION POINT IN A TWO-PREY ONE PREDATOR DYNAMICAL SYSTEM
}

\author{
Marwan $^{1}$, J.M. Tuwankotta, and E. Harjanto \\ Analysis and Geometry Research Group, \\ Faculty of Mathematics and Natural Sciences, Institut Teknologi Bandung, \\ Jl. Ganesha 10, Bandung, 40132. \\ ${ }^{1}$ Corresponding author: marwanmath@yahoo.co.id
}

\begin{abstract}
We propose an application of the classical Lagrange Multiplier method for computing fold bifurcation point of an equilibrium in a one-parameter family of dynamical systems. We have used the fact that an equilibrium of a system, geometrically can be seen as an intersection between nullcline manifolds of the system. Thus, we can view the problem of two collapsing equilibria as a constrained optimization problem, where one of the nullclines acts as the cost function while the other nullclines act as the constraints.
\end{abstract}

Key words and Phrases: Fold Bifurcation, Constrained Extremum, Dynamical Systems.

\begin{abstract}
Abstrak. Dalam paper ini kami menyajikan sebuah contoh penggunaan metode Pengali Lagrange dalam menghitung titik bifurkasi fold dari sebuah ekuilibrium pada keluarga satu parameter dari sistem dinamik. Kita menggunakan fakta bahwa titik ekuilibrium dari sistem dapat dipandang sebagai perpotongan dari permukaanpermukaan nullcline dari sistem dinamik terkait. Akibatnya, kita dapat memandang permasalahan bertemunya dua ekuilibria kemudian hilang, sebagai masalah optimisasi terkendala, dimana salah satu nullcline berfungsi sebagai fungsi biaya dan nullcline lainnya sebagai kendala.
\end{abstract}

Kata kunci: Bifurkasi Fold, Ekstremum Terkendala, Sistem Dinamik.

2000 Mathematics Subject Classification: 34C35; 34C23; 34A34. Received: 14-08-2017, revised: 07-02-2018, accepted: 08-02-2018. 


\section{INTRODUCTION}

The dynamics and bifurcations in a Predator-Prey type of dynamical systems have been studied extensively in the literature. Originated in the work of A.J. Lotka (1920) and V. Voltera (1926) (see [2]), the application of this model can be found in various field of sciences and engineering. To name but a few, in enzyme kinetics [3], in parasitology [8], in economy [4, 9], in atmospheric science [13], in optimization $([10,17]$, in molecular biology [1].

Fold bifurcation of equilibrium is a co-dimension one phenomena in the theory of bifurcations (see [14]). It occurs in a family of dynamical systems with one parameter (or more), already in low dimensional system (even in one dimensional dynamics). Qualitatively, this bifurcation described the phenomena of collapsing two equilibria, of different stability type, into one equilibrium of a degenerate type, and vanishes, as the bifurcation parameter varies through the bifurcation point in the parameter space.

In this paper we propose by means of an example, the application of the classical Lagrange Multiplier method for computing the fold bifurcation point. To our knowledge, some of the first applications of this method are found in [11] and [16]. However, the method has not been explained clearly there. In this paper we focus on how to apply the Lagrange Multiplier method to compute fold bifurcation point.

Computation for fold bifurcation point is usually done numerically by using the continuation software, for example AUTO2000 [6]. To do it analytically is quite involved, i.e. computing equilibrium depending on the bifurcation parameter, linearizing in the vicinity of that equilibrium, and then finding eigenvalues. Using the method we propose in this paper, the problem reduces to finding maximum or minimum of a constrained optimization problem which is part of a standard course in first year mathematics. Furthermore, the bifurcation parameter is not involved in the computation.

The dynamical system that we are dealing with is three dimensional with many parameters. Thus the complexity of the computation become significantly increasing in comparison with the two previously mentioned examples in the literature $([11,16])$. The system can be seen as a variant of a Two Prey One Predator model. Recently, interest on the Two Prey One Predator model has been increasing in the literature. See for example in $[7,12,18]$. However, as is mentioned earlier our focus in this paper is not on the dynamical property of the model it self. The model in this paper serves as a motivating example for applying this classical Lagrange Multiplier method to compute fold bifurcation point.

The paper is organized as follows. We start with presenting the Lagrange Multiplier method, and give a simple example that illustrate why this method can be used to compute fold bifurcation point. We then proceed with the Two Prey One Predator model and reformulate the system of equations that gives the equilibrium 
for the system as its solution. This system need to be reformulated as a constrained optimization problem. Applying the method there, we check the result by explicitly computing the eigenvalues each equilibria, to show that they are similar with the situation where we have fold bifurcation. The result is also compared with the result by using continuation software AUTO2000 [6].

\section{The Lagrange Multiplier Method}

Let $F$ and $G$ be real valued functions of two variables with continuous first partial derivatives on some open set containing the constraint curve $G(x, y)=0$ and assume that $\nabla G \neq 0$ any point on this curve. If $F$ has a constrained relative extremum, then this extremum occurs at a point $\left(x_{0}, y_{0}\right)$ on the constraint curve at which the gradient vectors $\nabla F\left(x_{0}, y_{0}\right)$ and $\nabla G\left(x_{0}, y_{0}\right)$ are parallel; that is, there is some number $\lambda$ such that

$$
\nabla F\left(x_{0}, y_{0}\right)=\lambda \nabla G\left(x_{0}, y_{0}\right) .
$$

Thus, the point $\left(x_{0}, y_{0}\right)$ where relative extremum of function $F$ occurs under the constraint $G=0$, is derived from the solution of system :

$$
\left\{\begin{array}{l}
\nabla F=\lambda \nabla G \\
G=0
\end{array}\right.
$$

In Figure 1 we have presented a geometric illustration of the situation where the constrained relative extremum of $F$ is achieved on the level set $G=0$. For the proof of this Lagrange Multiplier method, see [5] or other classical textbook on multivariable Calculus.

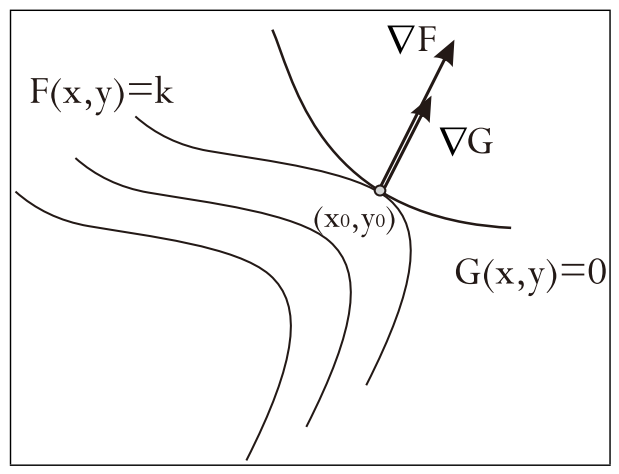

FIGURE 1. An illustration for Lagrange Multiplier method, where $G=0$ is the constraint, and $F$ is the cost function. Note that since we can take $-G$ as the constraint, then the direction of $\nabla G$ can be reversed without changing the result. 

function

Let us consider a very simple example. We want to find the extremum of the

$$
F(x, y)=3 x y \text { on the line: } G(x, y)=2 x+y-1=0 .
$$

Then, using the Lagrange Multiplier method, we derive a system of algebraic equations:

$$
\left\{\begin{array}{lll}
3 y-2 \lambda & = & 0 \\
3 x-\lambda & = & 0 \\
2 x+y-1 & = & 0
\end{array}\right.
$$

This system of equations can be solved easily; we have $\left(x_{0}, y_{0}\right)=(0.25,0.5)$ and $\lambda=0.75$. In Figure 2, we have presented the geometrical illustration for the situation. We have plotted three different level sets of $F$, i.e.: $F(x, y)=0.65$, $F(x, y)=0.375$, and $F(x, y)=0.15$.

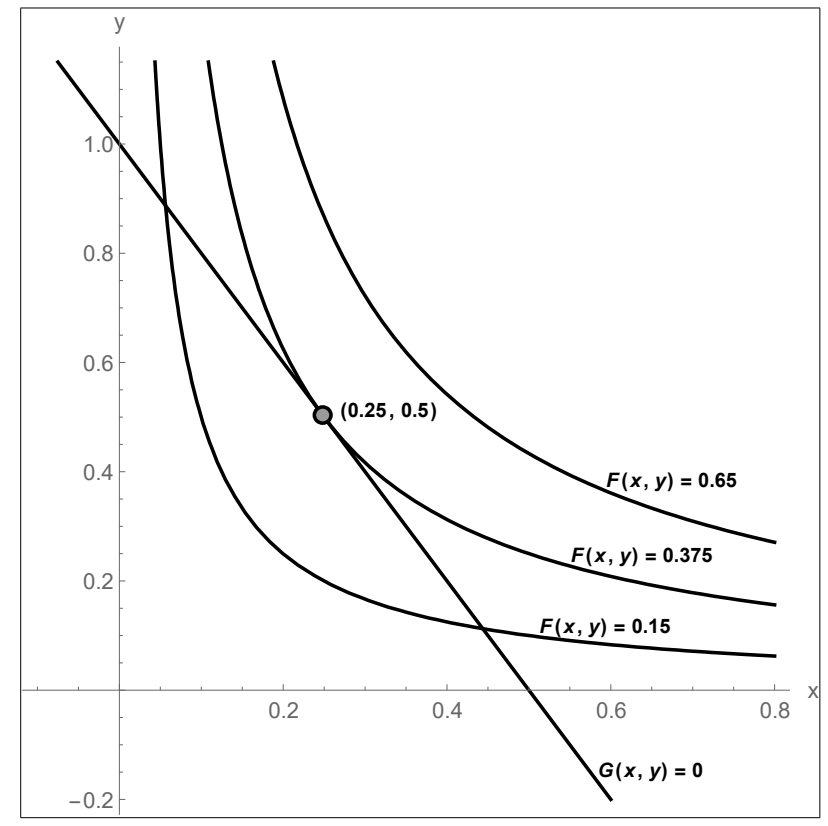

Figure 2. A simple example of how the Lagrange Multiplier method is applied to a constrained optimization problem (2). If the level set of $G$ and $F$ are both nullclines of a dynamical system, then each of the intersections corresponds to an equilibrium of that dynamical system.

Applications to computation of fold bifurcation point. Let us now consider a system of ordinary differential equations

$$
\begin{aligned}
& \dot{x}=f_{1}(x, y)-\alpha f_{2}(x, y) \\
& \dot{y}=G(x, y),
\end{aligned}
$$


where $f_{1}, f_{2}$ and $G$ are real valued smooth functions of $(x, y)$ The equilibrium of (3) can be seen as:

$$
F(x, y)=\frac{f_{1}(x, y)}{f_{2}(x, y)}=\alpha \text { and } G(x, y)=0 .
$$

We can still assume some smoothness of $F$ except in the neighbourhood of the zeros of $f_{2}$.

Let us assume that we have found a solution for the constrained optimization problem, of:

$$
\operatorname{Max} / \min F(x, y) \text { subjected to } G(x, y)=0 \text {. }
$$

We have to assume that the gradient vector of $G$ is non vanishing. In the trivial case, the vanishing gradient of $G$ corresponds to the case where the level set of $G$ consists of a single point. Another possibility is when the level set looses its smoothness, for example in the case where: $G(x, y)=x^{2}-y^{3}(1-y)$ at $(0,0)$.

Using the Lagrange multiplier method, we have $\left(x_{0}, y_{0}, \lambda_{0}\right)$ such that:

$$
\nabla F\left(x_{0}, y_{0}\right)=\lambda_{0} \nabla G\left(x_{0}, y_{0}\right) \text { and } G\left(x_{0}, y_{0}\right)=0 .
$$

We set $\alpha_{0}=F\left(x_{0}, y_{0}\right)$. Thus, the point $\left(x_{0}, y_{0}\right)$ corresponds to an equilibrium of (3) for $\alpha=\alpha_{0}$. Note that since

$$
f_{1}(x, y)-\alpha f_{2}(x, y)=f_{2}(x, y)(F(x, y)-\alpha),
$$

then linearizing $(3)$ in the neighbourhood of $\left(x_{0}, y_{0}\right)$ gives:

$$
\begin{aligned}
J & =\left.\left(\begin{array}{cc}
\frac{\partial f_{2}}{\partial x}\left(F-\alpha_{0}\right)+f_{2} \frac{\partial F}{\partial x} & \frac{\partial f_{2}}{\partial y}\left(F-\alpha_{0}\right)+f_{2} \frac{\partial F}{\partial y} \\
\frac{\partial G}{\partial x} & \frac{\partial G}{\partial y}
\end{array}\right)\right|_{\left(x_{0}, y_{0}\right)} \\
& =\left.\left(\begin{array}{cc}
f_{2} \frac{\partial F}{\partial x} & f_{2} \frac{\partial F}{\partial y} \\
\frac{\partial G}{\partial x} & \frac{\partial G}{\partial y}
\end{array}\right)\right|_{\left(x_{0}, y_{0}\right)},
\end{aligned}
$$

and since $\nabla F\left(x_{0}, y_{0}\right)=\lambda_{0} \nabla G\left(x_{0}, y_{0}\right)$ we have that the determinant of $J$ vanishes.

Proposition 2.1. The point $\left(x_{0}, y_{0}\right)$ corresponds to a degenerate equilibrium with exactly one zero eigenvalue, for $\alpha=\alpha_{0}=\frac{f_{1}\left(x_{0}, y_{0}\right)}{f_{2}\left(x_{0}, y_{0}\right)}$, assuming the trace of $J$ is non vanishing, or equivalently:

$$
\frac{\partial f_{1}}{\partial x}\left(x_{0}, y_{0}\right)-\alpha_{0} \frac{\partial f_{2}}{\partial x}\left(x_{0}, y_{0}\right)+\frac{\partial G}{\partial y}\left(x_{0}, y_{0}\right) \neq 0
$$

Proof. Since the determinant of $J$ is zero, then one of the eigenvalue is 0 and the other eigenvalue is the value of the trace of $J$.

We end this section with a proposition relating the maximum (or minimum) point of a constrained optimization problem with fold bifurcation point.

Proposition 2.2. If $\left(x_{0}, y_{0}, \lambda_{0}\right)$ is a maximum point of $F(x, y)$ subjected to $G(x, y)=$ 0 , then for $\alpha<\alpha_{0}=\frac{f_{1}\left(x_{0}, y_{0}\right)}{f_{2}\left(x_{0}, y_{0}\right)}$ the system (3) has two equilibria in the neighbourhood of $\left(x_{0}, y_{0}\right)$ in phase space. These equilibria coalesce into a degenerate equilibrium 
point $\left(x_{0}, y_{0}\right)$ as $\alpha=\alpha_{0}$ and disappear as $\alpha>\alpha_{0}$. The situation is reverse when $\left(x_{0}, y_{0}, \lambda_{0}\right)$ is a minimum point.

\section{The Two Prey One Predator model}

Let us consider a system of ordinary differential equations in $\mathbb{R}^{3}$, i.e.:

$$
\begin{aligned}
\dot{x} & =x-\frac{1}{k_{1}} x^{2}-\frac{\mu x z}{c x+1} \\
\dot{y} & =\alpha x-\beta y-\frac{1}{k_{2}} y^{2}-\frac{\eta y z}{a y^{2}+b y+1} \\
\dot{z} & =-\delta z+\frac{x z}{c x+1}+\frac{y z}{a y^{2}+b y+1} .
\end{aligned}
$$

Here, $x$ and $y$ represent the population density of prey while $z$ of predator's. In this model, we assume that the prey populations come from the same species but they are classified by their maturity: the young prey $(x)$ and the adult prey $(y)$. The parameter $\alpha$ is the rate of migration from the young prey class into the adult prey class. Parameters $\beta$ and $\delta$ measure the mortality rate of adult prey and predator, respectively. The predation factor is measured by the parameters $\mu$ and $\eta$. Parameters: $k_{1}$ and $k_{2}$ measure the carrying capacity of the environment with respect to the prey. We assume the value of all of these parameters are non negative real numbers.

We have included response functions that measures the predation as rational functions of the density of prey. For the young prey, the denominator of the response function is linear, while for the adult prey is quadratic. There are two parameters in the response function for the adult prey, $a$ and $b$. They control the saturation (a) and group defense mechanism $(b)$. These parameters can have negative value.

Let us consider the nullcline manifolds

$$
\begin{aligned}
& S_{1}: 0=1-\frac{1}{k_{1}} x-\frac{\mu z}{c x+1} \\
& S_{2}: 0=\alpha x-\beta y-\frac{1}{k_{2}} y^{2}-\frac{\eta y z}{a y^{2}+b y+1} \\
& S_{3}: 0=-\delta+\frac{x}{c x+1}+\frac{y}{a y^{2}+b y+1} .
\end{aligned}
$$

These manifolds are found by taking each of the components of the vector field in (4) to be zero. However we have ignored two other manifolds, i.e. one is defined by $x=0$ and the other is $z=0$, since we are interested on analyzing equilibrium at $x>0, y>0$ and $z>0$. Thus, we divide out the factor $x$ from the first equation and the factor $z$ from the third equation in (4)

Each of these nullclines defines a surface in $\mathbb{R}^{3}$. In Figure 3 we have presented a geometric illustration of these surfaces for some values of the parameter, i.e.: $\alpha=1, \beta=0.95, \delta=1, k_{1}=5, k_{2}=1.75, \mu=0.3, \eta=0.5, a=4.75, b=-2.5$, and $c=1$. There are two points in Figure 3 indicated by solid dots, which are the intersection between the three nullclines for $x>0, y>0$ and $z>0$. 


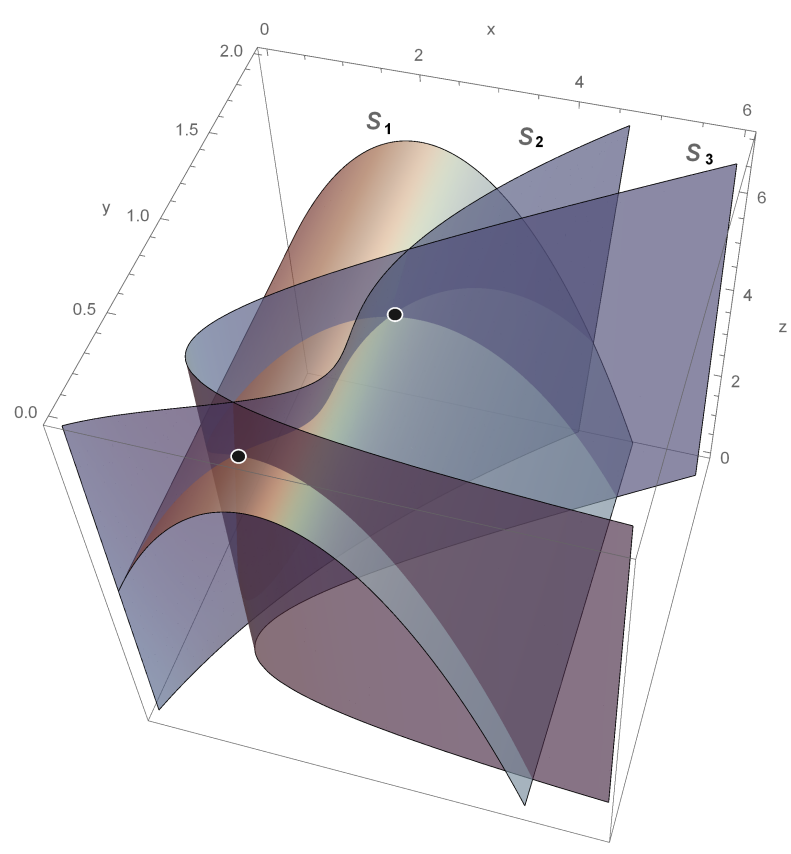

Figure 3. A graph of the surfaces $S_{1}, S_{2}, S_{3}$ for $\alpha=1, \beta=0.95$, $\delta=1, k_{1}=5, k_{2}=1.75, \mu=0.3, \eta=0.5, a=4.75, b=-2.5$, and $c=1$.

Consider the equation for $S_{1}$; we can solve this for $z$. Then we substitute the solution into the equation for $S_{2}$. By doing this, we reduce the problem into two dimensional problem:

$$
\begin{aligned}
& S_{4}:(x-p)^{2}-A(y-q)^{2}=r \\
& S_{3}:-\delta+\frac{x}{c x+1}+\frac{y}{a y^{2}+b y+1}=0
\end{aligned}
$$

where

$$
\begin{aligned}
p & =\frac{\eta k_{1}(c \delta-1)-k_{1} \mu \alpha-\eta \delta}{2 \eta(c \delta-1)}, \quad q=-\frac{\beta k_{2}}{2} \\
A & =\frac{k_{1} \mu}{\eta(c \delta-1) k_{2}}, \quad r=p^{2}-A q^{2}+\frac{k_{1} \delta}{c \delta-1} .
\end{aligned}
$$

Note that $S_{4}$ is a quadratic curve family, and in particular for $A<0$ and $r>0$ then $S_{4}$ is an ellipse. To formulate the system of equations as a constrained optimization problem we choose $S_{4}$ as the constrained, and reformulate $S_{3}$ into the cost function (i.e. function that is being optimized). There are four parameters i.e. 
$a, b, c$ and $\delta$, so that we can have four options for the cost function, namely:

$$
\begin{aligned}
& F_{\delta}(x, y)=\frac{x}{c x+1}+\frac{y}{a y^{2}+b y+1} \\
& F_{a}(x, y)=\left(\frac{c x+1}{x(c \delta-1)+\delta}-\frac{1}{y}-b\right) \frac{1}{y} \\
& F_{b}(x, y)=-a y+\frac{c x+1}{x(c \delta-1)+\delta}-\frac{1}{y}, \text { or } \\
& F_{c}(x, y)=\frac{a y^{2}+b y+1}{\left(a y^{2}+b y+1\right) \delta-y}-\frac{1}{x} .
\end{aligned}
$$

Thus, writing

$$
G(x, y)=(x-p)^{2}-A(y-q)^{2}-r
$$

the fold bifurcation point is found by solving the system of equations:

$$
\left\{\begin{array}{l}
\nabla F_{\nu}=\lambda \nabla G \\
G=0
\end{array}\right.
$$

where: $\nu \in\{a, b, c, \delta\}$.

However, the computation of a solution for the system (8) in its full generality is still too cumbersome due to number of the parameters involved. To simplify, we fix some values for the parameters, i.e.: $\alpha=0.8, a=7.29, \beta=0.75, b=-2.59$, $c=1, \delta=0.8, \eta=0.25, k_{1}=6, k_{2}=4$, and $\mu=0.75$. Clearly, if we choose $F_{a}$ as the cost function, then we let $a$ as a free parameter, and similarly for the other choices. In this paper, we will only do the case for $F_{a}$ and $F_{b}$.

Remark 3.1. Note that from (7) it is clear that the parameter $p, A$, and $r$ in the definition of $G$ depends on other parameter, which might be the bifurcation parameters: $\delta$ or $c$. Thus, if we choose for $F_{\delta}$ or $F_{c}$ as the cost function, we have to choose the value of the parameter in such a way such that $p, A$ and $r$ are constant.

\section{Numerical Results}

In this section, we present two examples of numerical computation of the fold bifurcation point in system (8) using the Lagrange Multiplier method, i.e. using $F_{b}$ and $F_{a}$ as the cost functions. The case where the cost function is $F_{b}$ will be done in details while the other case will be summarized at the end of the section.

The case where $b$ is the bifurcation parameter. Using the above listed value of the parameters in the previous section, we derive two equilibria at which fold bifurcation occurs respectively i.e. $\left(x_{0}, y_{0}\right)=(1.59866,1.12602)$ and $\left(x_{0}, y_{0}\right)=$ $(0.736391,0.460349)$. These results are shown in Figure 4 . The $z_{0}$-component of these points can be computed by substituting them into $S_{1}$ in system (5) to have the equilibria in $\mathbb{R}^{3}$ as follows: 


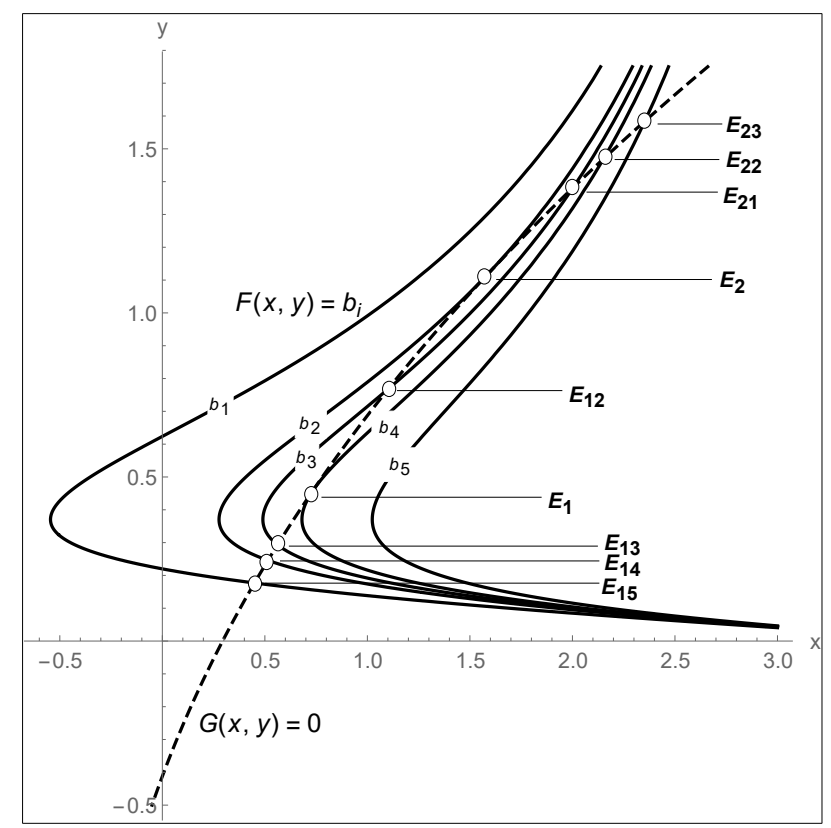

FiguRE 4. Graph of the intersection between $F$ and $G$ as the parameter $b$ varies from left to right $b_{1}=-5, b_{2}=-3.68591$, $b_{3}=-3.2589, b_{4}=-2.86798$ and $b_{5}=-2$.

$$
\begin{aligned}
& E_{1}=\left(x_{0}, y_{0}, z_{0}\right)=(1.59866,1.12602,2.54168) \\
& E_{2}=\left(x_{0}, y_{0}, z_{0}\right)=(0.736391,0.460349,2.03104)
\end{aligned}
$$

Furthermore, the fold bifurcation point (in parameter space) is computed by substituting $E_{1}$ and $E_{2}$ into the cost function $F_{b}(x, y)$, to derive:

$$
\begin{aligned}
& b_{2}=F_{b}(1.59866,1.12602)=-3.68591 \\
& b_{4}=F_{b}(0.736391,0.460349)=-2.86798
\end{aligned}
$$

These results are checked by substituting the coordinate of each of the equilibria into the system (4). Let us now look at Figure 4 . The level set of $F_{b}(x, y)=$ $b_{1}=-5$ is the curve on the most left from the family of curves $F_{b}(x, y)=b$ which are plotted using thickened lines. The curve intersects the curve $G(x, y)=0$ which is plotted using dashed line, at one point only: $E_{15}$. As we increases the value of $b$ to $b_{2}=-3.68591$, we have the curve $F(x, y)=b_{2}$, which is the second curve from the left in Figure 4. This curve intersects $G(x, y)=0$, transversally at the point labelled as $E_{14}$ and non transversally at the point labelled as $E_{2}$, which is one of the solutions of (8) (see also the formula in (9) and (10)). As we increase the value of $b$ further to $b_{3}=-3.2589$, we are on the third curve from the left of the family $F(x, y)=b$. This curve intersect $G(x, y)=0$ at three points, labelled 
as: $E_{13}, E_{12}$ and $E_{21}$. Similarly, the intersection between $F(x, y)=b_{4}=-2.86798$ and $G(x, y)=0$, gives us equilibria: $E_{1}$ and $E_{22}$, while the intersection between $F(x, y)=b_{5}=-2$ and $G(x, y)=0$, gives the equilibrium: $E_{23}$.

In Table 1 we have listed the coordinate of all of the equilibria found by varying $b$ sequently : $b_{1} \longmapsto b_{2} \longmapsto b_{3} \longmapsto b_{4} \longmapsto b_{5}$. As we can see from the Table 1 , there is a change of stability between $E_{15}$ to $E_{14}$. The explanation for this is due to the occurrence of Hopf bifurcation which cannot be explained by the method we propose in this paper. However, the eigenvalues for $E_{2}, E_{12}$ and $E_{21}$ give a strong indication that indeed fold bifurcation occurs as the parameter $b$ passes through: -3.68591 . Similarly, as $b$ passes through: -2.86798 we have another strong indication that fold bifurcation occurs.

\begin{tabular}{|c|c|c|c|}
\hline$b$ & Equilibrium & $(x, y, z)$ & Eigenvalues of Jacobian System \\
\hline \hline-5 & $E_{15}$ & $(0.44,0.17,1.78)$ & $\lambda_{1,2}=-0.013 \pm 0.85 i, \lambda_{3}=-3.44$ \\
\hline-3.6859 & $E_{14}$ & $(0.51,0.24,1.84)$ & $\lambda_{1,2}=0.009 \pm 0.55 i, \lambda_{3}=-1.76$ \\
& $E_{2}$ & $(1.59,1.12,2.54)$ & $\lambda_{1}=0.065, \lambda_{2}=0, \lambda_{3}=-1.05$ \\
\hline-3.2589 & $E_{13}$ & $(0.56,0.29,1.88)$ & $\lambda_{1,2}=0.013 \pm 0.65 i, \lambda_{3}=-1.35$ \\
& $E_{21}$ & $(2.01,1.38,2.66)$ & $\lambda_{1,2}=0.026 \pm 0.23 i, \lambda_{3}=-1.31$ \\
& $E_{12}$ & $(1.08,0.75,2.28)$ & $\lambda_{1}=0.374, \lambda_{2,3}=-0.503 \pm 0.20 i$ \\
\hline-2.8679 & $E_{22}$ & $(2.15,1.46,2.69)$ & $\lambda_{1,2}=0.018 \pm 0.26 i, \lambda_{3}=-1.37$ \\
& $E_{1}$ & $(0.73,0.46,2.03)$ & $\lambda_{1}=0.084, \lambda_{2}=0, \lambda_{3}=-0.63$ \\
\hline-2 & $E_{23}$ & $(2.35,1.58,2.71)$ & $\lambda_{1,2}=0.002 \pm 0.02 i, \lambda_{3}=-1.46$ \\
\hline
\end{tabular}

TABLE 1. Variation of the real part of the eigenvalues of the Jacobian of system (4)

As a comparison, we also present the computation of fold bifurcations using pseudo-arclength continuation technique (see [6]). The bifurcation diagram computed using this method is plotted in Figure 5. It is evident that the two fold bifurcations occur at $b_{0}=-2.867978$ and at $b_{0}=-3.685906$, which match the result using Lagrange Multiplier method. Similarly, for the other choice of cost function, i.e. $F_{a}$, have found fold bifurcations for $a=6.70936$, at $(0.779315,0.499127,2.06428)$, and $a=6.25962$ at $(1.40171,0.990878,2.45417)$. 


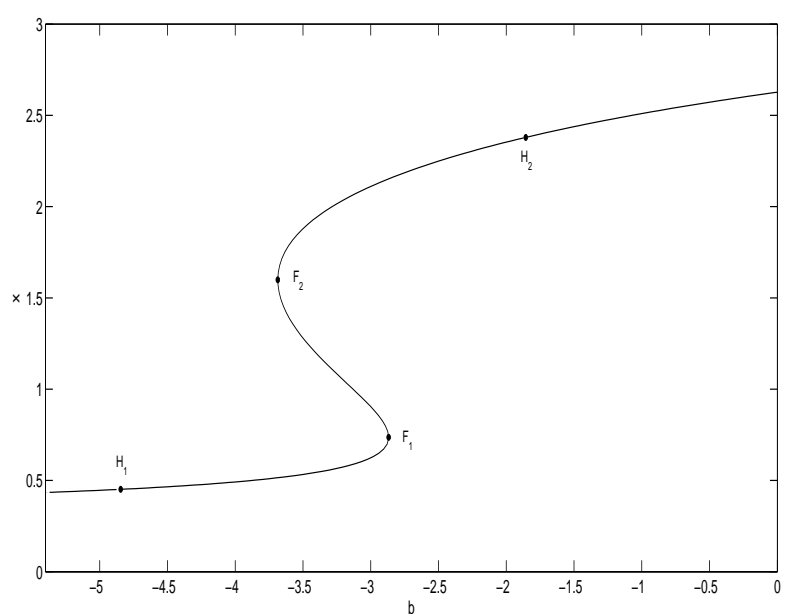

FiguRE 5. A curve of equilibria in $(b, x)$-plane which indicates two fold bifurcation points $\left(F_{1}\right.$ and $\left.F_{2}\right)$ as the parameter $b$ varies

\section{Concluding Remarks}

We have proposed an application of Lagrange Multiplier method in finding fold bifurcation point for two dimensional system of ordinary differential equations. For our example of Two Prey One Predator model, the number of parameters in the system is quite large so that we decided to fixed arbitrary value for all but one of the parameters in the system. The result is then compared to the one using the continuation software AUTO2000 [6] and shows a remarkable agreement between the two results.

To prove that the extremum of a constrained optimization problem indeed corresponds to a fold bifurcation in the dynamical systems, is a subject of future investigation. What we have done in this paper is to show that geometrically what happen in a neighbourhood of a maximum (or minimum) point of a constraint optimization problem is similar to the one in the neighbourhood of fold bifurcation in phase space. Furthermore, it is interesting to extend our observation to higher dimensional case. What we mean by this is applying the Lagrange multiplier method with more than one constraint, rather than reducing the problem to two dimensional setting.

Acknowledgement. This research is mainly supported by Riset ITB (FMIPAPN-6-42-2017: Bifurkasi Cusp-Bogdanov-Takens pada Sistem Dinamik PredatorPrey; P.I.: J.M. Tuwankotta). JMT thanks also Riset P3MI FMIPA ITB for financial support for visiting TU Delft in December 2017, at where a part of this research has been done. He also thanks Ferdinand Verhulst (Utrecht University) and Wim van Horssen (TU Delft) for their hospitality during the visit. Marwan 
thanks BPP-DN Dikti for financial support for his research at ITB.

\section{REFERENCES}

[1] F. K. Balagaddé, H. Song, J. Ozaki, C.H. Collins, M. Barnet, F.H. Arnold, S.R. Quake, and L. You, A synthetic Escherichia coli predatorprey ecosystem, Mol. Syst. Biol. (2008), 4:187.

[2] A.A. Berryman, The origins and evolution of predator-prey theory, Ecology, 73(5),(1992), 15301535.

[3] Briggs, G. E., and Haldane, J. B. A Note on the Kinetics of Enzyme Action, Biochem J 19, (1925) pp. 338-339.

[4] Z. Cai, Q. Wang, and G. Lie, Modeling the Natural Capital Investment on Tourism Industry Using a Predator-Prey Model, in Advances in Computer Science and its Applications, Vol. 279 of the series Lecture Notes in Electrical Engineering (2014) pp 751-756.

[5] Dineen, S. Multivariate Calculus and Geometry, third edition, Springer Undergraduate Mathematics Series, Springer (2014), London etc.

[6] Doedel, E. J., et al., 2000, AUTO: Continuation and Bifurcation Software for Ordinary Differential Equations (with HomCont), California Institute of Technology, Pasadena, California.

[7] Elettreby, M.F., Two-prey one-predator model Chaos, Solitons \& Fractals 39 (5) (2009), Pp. 2018-2027

[8] A. Fenton, and S.E. Perkins, Applying predator-prey theory to modeling immune-mediated, within-host interspecific parasite interactions, Parasitology 137(6) (2010 May): 1027-38.

[9] R.M. Goodwin, A Growth Cycle, in Feinstein, C. H. (ed), in Socialism, Capitalism, and Economic Growth, Cambridge, Cambridge University Press, (1967), pp. 54-58.

[10] C. Grimme, and J. Lepping, Integrating niching into the predator-prey model using epsilonconstraints, Proceedings of the 13th Annual Conference Companion on Genetic and Evolutionary Computation (GECCO'11) (2011): 109-110.

[11] Harjanto, E., and Tuwankotta, J.M., Bifurcation of Periodic Solution in a Predator-Prey Type of Systems with Non-monotonic Response Function and Periodic Perturbation, International Journal of Non-Linear Mechanics, 85, 2016, 188-196.

[12] Klebanoff, A., Hastings, A., Chaos in one-predator, two-prey models: General results from bifurcation theory Mathematical Biosciences, 122(2), 1994, pp. 221-233

[13] Koren, I., Feingold, G., Aerosolcloudprecipitation system as a predator-prey problem., Proceedings of the National Academy of Sciences 108, 30 (2011): pp. 12227-12232.

[14] Kuznetsov, Y. A., 1998, Elements of Applied Bifurcation Theory, 2nd ed., Springer, New York.

[15] Lessard, J., Rigorous verification of saddlenode bifurcations in ODEs, Indagationes Mathematicae, 27, 2016, 1013-1026.

[16] Owen, L., and Tuwankotta, J.M., Bogdanov-Takens Bifurcations in Three Coupled Oscillators System with Energy Preserving Nonlinearity, J. Indones. Math. Soc., 18(2), 2012, 73-83.

[17] A. Sharma, and, N. Singh, Object detection in image using predator-prey optimization, Signal \& Image Processing: An International Journal (SIPIJ), vol. 2 (1)(2011), 205-221.

[18] Tripathi, J. P., Syed Abbas, S., Manoj Thakur, M., Local and global stability analysis of a two prey one predator model with help Communications in Nonlinear Science and Numerical Simulation, 2014, pp. 3284-3297 